OPEN ACCESS

Edited by:

Stacy T. Sims,

Auckland University of Technology,

New Zealand

Reviewed by:

Jonathan Peake,

Queensland University of

Technology, Australia

William Conkright,

Madigan Army Medical Center,

United States

*Correspondence:

David C. Nieman

niemandc@appstate.edu

Specialty section: This article was submitted to

Sport and Exercise Nutrition,

a section of the journal

Frontiers in Nutrition

Received: 16 October 2021 Accepted: 24 November 2021 Published: 14 December 2021

Citation:

Nieman DC (2021) Multiomics Approach to Precision Sports

Nutrition: Limits, Challenges, and Possibilities. Front. Nutr. 8:796360.

doi: 10.3389/fnut.2021.796360

\section{Multiomics Approach to Precision Sports Nutrition: Limits, Challenges, and Possibilities}

\section{David C. Nieman*}

North Carolina Research Campus, Human Performance Laboratory, Department of Biology, Appalachian State University, Boone, NC, United States

Most sports nutrition guidelines are based on group average responses and professional opinion. Precision nutrition for athletes aims to improve the individualization of nutrition practices to optimize long-term performance and health. This is a 2-step process that first involves the acquisition of individual-specific, science-based information using a variety of sources including lifestyle and medical histories, dietary assessment, physiological assessments from the performance lab and wearable sensors, and multiomics data from blood, urine, saliva, and stool samples. The second step consists of the delivery of science-based nutrition advice, behavior change support, and the monitoring of health and performance efficacy and benefits relative to cost. Individuals vary widely in the way they respond to exercise and nutritional interventions, and understanding why this metabolic heterogeneity exists is critical for further advances in precision nutrition. Another major challenge is the development of evidence-based individualized nutrition recommendations that are embraced and efficacious for athletes seeking the most effective enhancement of performance, metabolic recovery, and health. At this time precision sports nutrition is an emerging discipline that will require continued technological and scientific advances before this approach becomes accurate and practical for athletes and fitness enthusiasts at the small group or individual level. The costs and scientific challenges appear formidable, but what is already being achieved today in precision nutrition through multiomics and sensor technology seemed impossible just two decades ago.

Keywords: exercise, nutrition, genomics, metabolomics, proteomics, carbohydrates, polyphenols, immunity

\section{PERSONALIZED AND PRECISION NUTRITION}

A core tenet of the 2020-2025 Dietary Guidelines for Americans (DGAs) is that a healthy dietary pattern is a customizable framework that supports tailored individual choices to meet personal preferences (1). The DGAs have also been characterized, however, as population-level recommendations that do not provide ideal nutrition guidance for each individual (2). Individuals vary widely in their metabolic responses to specific components of the DGAs, and most of this variance is unexplained $(3,4)$. The National Institutes of Health $(\mathrm{NIH})$ and other agencies are focused on investigations to improve scientific understanding of these individual differences, adding momentum to the paradigm shift toward personalized nutrition $(5,6)$. This narrative-style 
mini-review outlines the limits, challenges, and possibilities of personalized nutrition, with a focus on multiomics approaches and how these can be applied to sport and exercise nutrition.

Personalized nutrition is expected to grow from $\$ 8.2$ billion in 2020 to $\$ 16.4$ billion by 2025 (7). This growth is being driven by increasing health and fitness awareness by consumers, dramatic drops in genotyping costs, access to free public archives of genetic variation data, digital healthcare, direct-to-consumer (DTC) kits, widespread availability and use of smartphone apps, consumer demand for supplements, and an aging population $(7,8)$. Advocates also urge that personalized nutrition compared to traditional approaches will be more effective in motivating individuals to improve their dietary intake and thereby mitigate disease risk factors $(9,10)$.

Several definitions for personalized nutrition have been proposed $(10,11)$. The International Life Sciences Institute emphasized that personalized nutrition for the generally healthy population should use "individual-specific information, founded in evidence-based science, to promote dietary behavior change that may result in measurable health benefits" (2). Others prefer the term precision nutrition that is focused on a systems biology and multiomics approach (i.e., using the tools of genomics, transcriptomics, proteomics, metabolomics, microbiomics, epigenetics) with integration bioinformatics and machine learning to sharpen the scientific certainty needed for specific nutrition recommendations $(3,11)$.

Personalized and precision nutrition are emerging disciplines that aim to stratify individuals into ever-smaller groups as the science develops and specific nutrition guidance can be conveyed accurately $(7,11)$. There are many challenges to overcome before personalized and precision nutrition becomes an accepted component of nutrition science and professional practice. Metabolic heterogeneity with high individual-toindividual variance is largely unexplained (4). Precision nutrition aims to improve scientific understanding of responders and nonresponders to dietary interventions. This process will require a tremendous investment by multiomics-focused investigators and funding agencies, with strong bioinformatics support (11, 13). Once metabolic heterogeneity is better understood, the findings must next be translated to accurate dietary advice that is efficacious and health-promoting $(6,11)$. The entire process will have to be linked to health behavior change support and research to determine if people are motivated enough to change and accept this costly and complex approach over the long term.

We are currently in a transition period with the rapid expansion of nutrition-based multiomics data but a lack of well-designed studies to demonstrate efficacious dietary recommendations at the small group or individual level (12). Humans have more than 21,000 genes, and each person has more than 50,000 single nucleotide polymorphisms (SNPs) $(3,13)$. Observed phenotypes are impacted by many genes, SNPs and other types of genetic variants, and epigenetic changes from environmental and lifestyle factors that influence the way genes work (13). People vary widely in how they respond to plant food bioactives and phytochemicals, in part due to differences in absorption, distribution, metabolism, and excretion (ADME) (6). As a result, inter-individual variation is considerable and far exceeds intra-individual variation in most multiomics studies
(4, 12). For example, even among twins, gut microbiome alpha diversity (richness) varies more over time between the twins than within a twin (14). All of this complicates the translation of genomics and other multiomics data into dietary recommendations for small groups and individuals (13).

A new generation of studies is needed with in depth phenotyping and integration of multiomics data with machine learning (a subbranch of Artificial Intelligence) to aid in the development of predictive precision nutrition models $(6,11,15)$. Supervised and unsupervised machine learning algorithms focus on patterns within large and complex precision nutrition datasets to develop maximum likelihood predictions about the outcomes of interest (15). The use of machine learning in precision nutrition is an emerging discipline, and one of the fundamental challenges is the development of high-quality datasets from large cohorts from which pertinent measurements have been obtained. Another challenge is the use of evaluation metrics to verify the actual effectiveness of the prediction models (15).

Decades of research on the genetic risk for obesity can serve as a lesson for the challenges that lie ahead in precision nutrition. The genetic component of BMI in the population accounts for about 40 to $50 \%$ of its variance after adjustment for age and sex, providing room for modifying effects of genetic variation to be assessed (16). It is now apparent from genome-wide association studies (GWAS) combined with large SNPs panels that obesity genetic risk is shaped by hundreds, perhaps thousands of DNA variants (16). As a result, no genetically based clinical screening algorithm has attained the predictive power needed to calculate obesity risk for individuals (16). The most important message from obesity genetics research is that people do not all have the same proneness to becoming obese and despite decades of effort this still remains difficult to predict.

What does this mean for precision nutrition? Both obesity and the way people respond to dietary interventions are impacted by many intrinsic and extrinsic factors. Genomics is just one of many components to measure and consider. Novel precision nutrition programs to manage obesity have been designed with personalized macronutrient compositions that vary based on the individual's genotype, enterotype, and other related factors (17). To refine this approach, comprehensive data sets from large groups are needed that include demographics, anthropometry, diet intake, physical activity, genomics, transcriptomics, epigenetics, proteomics, metabolomics, and environmental exposure $(3,11,12)$. With machine learning, these types of data sets can be modeled to improve understanding of interindividual variation and the development of more accurate precision nutrition recommendations. Although remarkable progress has been made, multiomics-based solutions and elucidations remain a work in progress, with high expectations that this will be a successful, albeit costly initiative $(6,13)$.

\section{PRECISION NUTRITION FOR ATHLETES AND PHYSICALLY ACTIVE INDIVIDUALS}

So what insights can be applied from precision nutrition to athletes and physically active individuals? Athletes vary widely in their lifestyle habits and metabolic and physiological responses 
to sports foods and supplements $(18,19)$. Most sports nutrition guideline statements and periodized nutrition plans are based on group average responses and professional opinion (20-22). Several organizations have recommended a biology systemsbased approach to adapt sports nutrition guidelines for a more personalized approach (19-23).

Figure 1 summarizes a precision nutrition approach for athletes. This is a 2-step process that focuses on collecting as much individual-specific, science-based information as possible on the individual, and then developing and delivering individualized nutrition guidance while monitoring and measuring health and performance outcomes. The first step emphasizes the acquisition of information using a variety of sources including lifestyle and medical histories, dietary assessment, physiological assessments from the performance lab and wearable sensors, and multiomics data from blood, urine, saliva, and stool samples. Depending on resources and during this transition period of continued investigation by scientists, this step may involve just a few targeted biomarker data with high effect sizes. The second step involves the delivery of science-based nutrition advice, behavior change support, and the monitoring of health and performance efficacy and benefits relative to cost. The sports nutrition precision approach is an emerging science with years of additional studies of large groups needed to ensure accurate, practical, and individualized nutrition guidance.

Despite scientific reservations at this point in time, the precision sports performance and nutrition DTC companies have already moved forward into the marketplace. A growing number of DTC companies gather pertinent demographics and lifestyle data, extract DNA from consumer's saliva samples using home kits, assess the presence or absence of genetic variants, predict inherent athletic ability, and/or provide individualized recommendations on exercise training and diet intake to improve performance $(8,24)$. Bloodwork and DNA data are also used to individualize guidelines for building muscle, reducing inflammation, boosting energy, optimizing mood, raising metabolism, improving cognition, and maintaining bone health.

The current consensus among genetics researchers is that DTC genetic tests are unable to provide accurate information regarding early athletic talent, training recommendations to maximize performance, or how to lose fat and build muscle (25, 26). Often the DTC genetic tests focus on polymorphisms for just a few genes (e.g., alpha-actinin-3 or ACTN3, and angiotensinconverting enzyme or ACE) despite the fact that there is no consistent scientific evidence that they have a sizeable influence on complex attributes such as athletic performance $(8,24-26)$. Hereditability estimates for cardiorespiratory fitness or VO2max are relatively high (about 50\%) $(27,28)$. Nonetheless, studies linking genotype and SNPs to cardiorespiratory fitness and other related attributes such as exercise training responses and exercise-induced changes in cardiometabolic risk factors have failed to produce definitive panels that could be used by DTC companies $(27,29-31)$. The use of global metabolomics and proteomics profiling as correlates of cardiorespiratory fitness has only recently been explored and these are best described as preliminary studies $(32,33)$.

Common nutrition-related traits that are assessed by DTC companies include food intolerance (e.g., lactose intolerance), food sensitivities (i.e., caffeine sensitivity), macronutrients (e.g., lipid metabolism), micronutrient metabolism (e.g., vitamins D and $\mathrm{C}$ metabolism), eating behavior (e.g., weight management), and oxidative stress (antioxidant and detoxifying capacity) (8). DTC companies seldom provide information on the specific genetic variants that are being assessed, and when listed, are often unrelated to the trait (8). A growing number of gene-nutrient interactions that may influence sport performance have been proposed, but in general, there is a dearth of strong research data at this time to make recommendations for athletes based on nutrigenomics (31).

Caffeine is one of just five evidence-based dietary supplements linked to performance optimization $(18,21)$, and serves as a prime example of the challenges in sport nutrigenomics. Studies generally support caffeine ingestion (3-13 mg/kg) for improved performance in a broad range of exercise modes, but individual responses vary widely (34). The cytochrome P450 1A2 (CYP1A2) gene produces an enzyme that metabolizes caffeine, but more than 13 SNPs can modify this gene's metabolic activity (35). One of the CYP1A2 SNPs is rs762551, and A/C and C/C carriers (54\% of population) experience decreased caffeine metabolism activity in comparison to more rapid metabolism in A/A carriers (46\% of population). Although CYP1A2 polymorphism has been hypothesized to alter caffeine's ergogenic effect, studies thus far are mixed, and CYP1A2 genotyping as a service to athletes is not recommended until more is known $(35,36)$.

Another interesting nutrient that serves as an example of the challenges that lie ahead in sport nutrigenomics is choline. Choline is an essential nutrient involved in multiple biochemical pathways related to performance including acetylcholine production for neurotransmission and phosphatidylcholine formation in muscle membranes $(37,38)$. Low dietary intake of choline can diminish concentrations of phosphatidylcholine in muscle cell membranes, making them more fragile and prone to exercise-induced muscle damage and leakage of creatine phosphokinase (CPK) (39). Common polymorphisms of genes involved with choline metabolism (about $10 \%$ of the population) include SNPs (rs2771040, rs1557502) associated with the solute carrier 44 choline transporter member 1 (SLC44A1) and choline kinase $(\mathrm{CHKB})$ genes $(38,39)$. People who carry these SNPs develop extremely high serum creatine phosphokinase (CPK) levels after stressful exercise bouts that include eccentric contractions (e.g., downhill running). These individuals may also develop rhabdomyolysis when dietary patterns low in choline are combined with stressful levels of exercise training. Some advocate widespread SNP screening to identify these individuals combined with recommendations to increase choline (e.g., more eggs and liver) and methyl-folate intake to mitigate exercise-induced muscle damage (39-41). However, definitive research to support this strategy is lacking, and long-term health and performance effects of carrying these gene polymorphisms are unknown. Studies have consistently failed to demonstrate 


\section{Precision Nutrition for Athletes Goal: Improved tailoring of nutritional practices to optimize both health and performance}

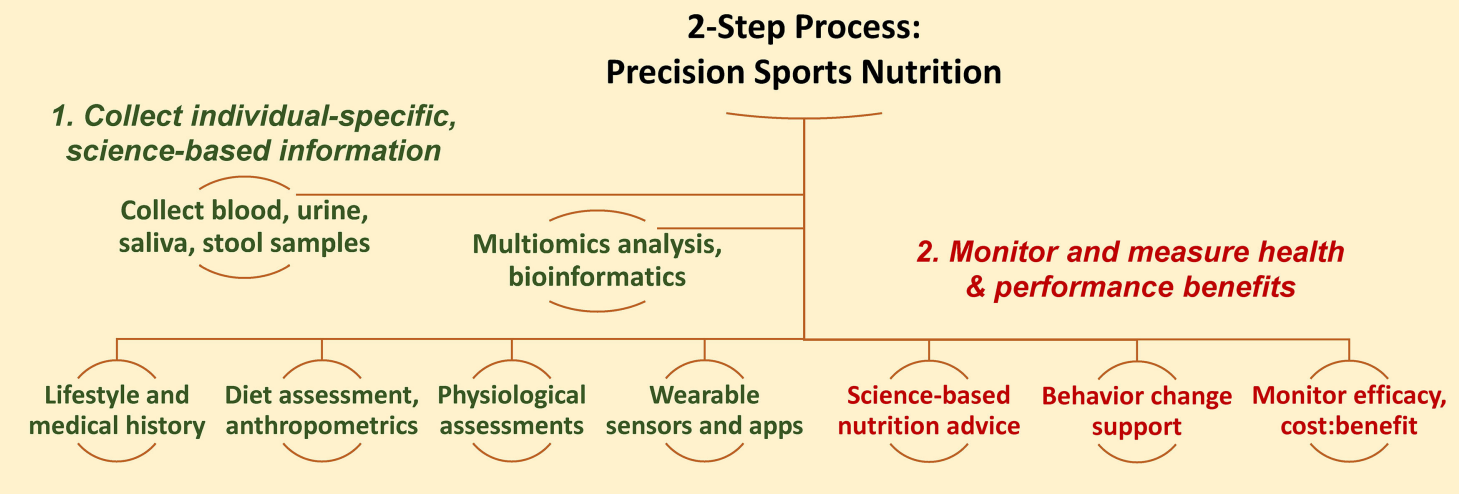

FIGURE 1 | Precision nutrition for athletes aims to improve tailoring of nutrition practices to optimize both health and performance. This is a 2-step process that emphasizes 1) the acquisition of individual-specific, science-based information using a variety of sources and 2) delivery of science-based nutrition advice with behavior change support and monitoring of efficacy and benefits relative to cost.

that supplemental choline has a positive influence on physical or cognitive performance $(42,43)$.

Precision nutrition relies on what can be accurately assessed at the individual level, and this process often begins and is ultimately limited with assessment of dietary intake and physical activity. The IOC recommends that a complete nutritional assessment of the athlete's diet should be undertaken before decisions regarding supplement use are made (18). Data obtained from dietary intake assessments (e.g., 24-h recall, food frequency questionnaires, dietary records) have limited utility because they are burdensome and based on selfreport, memory, social desirability reporting bias, and behavior change reactivity to monitoring $(44,45)$. Currently, physical activity compared to dietary intake monitoring tools are more advanced (44). Technological advances in wearable sensors have improved physiological and performance monitoring of mental and physical stress, sleep quality, blood glucose levels, body temperature, hydration status, oxygen consumption, heart rate, blood pressure, and exercise workloads (46). The use of wearable sensors facilitates continuous and real-time tracking of targeted outcomes that should strengthen the usefulness of nutrition guidance down to the individual level. The use of multimodal wearable sensors and miniature cameras in the area of nutritional assessment is expanding rapidly $(44,45,47)$. The ultimate goal is to make physical activity, dietary intake, and physiological monitoring seamless, passive, accessible, and accurate, and this will transform the effectiveness of precision nutrition (46-48).

\section{SYSTEMS BIOLOGY APPROACH TO PRECISION SPORTS NUTRITION}

Vigorous acute exercise bouts increase body metabolic demands 6 to 20 times above resting levels, and have profound, transitory effects on gene expression and blood/tissue levels of numerous metabolites, lipid mediators, and proteins (4959). These exercise-induced molecular changes are complex and incompletely understood. Human systems biology approaches with integrated multiomics profiling have been initiated to improve scientific understanding of the molecular underpinnings of related health and disease prevention benefits (50).

Multiomics profiling in exercise science has expanded rapidly due to the development of new technologies that provide simultaneous measurement of hundreds and thousands of molecules from small amounts of body fluids, cells, and tissues. Recent studies have linked acute vigorous exercise to changes in about 6,000 transcripts (from RNA sequencing), more than 300 proteins, and 300 to 700 metabolites (4956). Integrated molecular profiling during recovery from vigorous exercise indicates that multiple biological processes are affected including energy metabolism, oxidative stress, immune function and inflammation, tissue repair and remodeling, signaling pathways, cell growth and mobility, cardiovascular signaling and angiogenesis, and apoptosis (4952). Exercise proteomics has shown that hundreds of proteins are secreted by the muscle and other tissues discretely or within extracellular vesicles to regulate physiological processes 
throughout the body $(54,55,60)$. Exercise metabolomics has established that following intensive bouts lasting more than two hours, large-fold changes in numerous and diverse lipid-related metabolites occur reaching their nadir within a few hours with abatement after one day of recovery $(53,58)$. Other exercise-induced plasma metabolite shifts include a variety of amino acids and tricarboxylic acid (TCA) cycle intermediates including malate, aconitate, citrate, fumarate, succinate, and alpha-ketoglutarate. Postexercise changes in amino acids support metabolic requirements, and increases in TCA metabolites facilitate regulation of inflammation and immune function $(50,51,53,56)$. Intensive and prolonged exercise also increases plasma and muscle levels of 50 to 100 bioactive oxidation products from polyunsaturated fatty acids called oxylipins (57). Oxylipins have vital regulatory roles in many physiological processes including immune function, inflammation, cardiac and vascular function, and blood coagulation (57).

Thus, thousands of molecules are transiently affected by acute exercise, but the complex interplay between these molecules, the wide range of responses measured between individuals, and the use of relatively small sample sizes have limited scientific consensus (49-52). Fewer molecules differentiate exercise trained and untrained states compared to the much larger but transient shifts in proteins and metabolites following acute exercise bouts, and there is little overlap (49-52). Thus, a summation effect from regular acute exercise-induced shifts in gene expression and thousands of proteins and metabolites may play a larger role in mediating health effects than with chronic adaptations. The Molecular Transducers of Physical Activity Consortium (MoTrPAC) was established through an $\mathrm{NIH}$ Common Fund program to expand the science in this area and generate a molecular map of acute and chronic exercise training (61).

The application of multiomics approaches to sports nutrition is still an emerging area of scientific endeavor. Global and targeted metabolomics (with lipidomics) and proteomics improves the capacity to capture the complex biochemical effects resulting from a nutritional intervention with athletes during an exercise bout (62). Seminal studies in this area indicate that postexercise increases in lipidrelated metabolites, oxylipins, and inflammatory cytokines after hours of intensive cycling are strongly mitigated when overnight-fasted athletes ingest carbohydrate compared to water only (62-65). Exercising under "low-carbohydrate" availability is physiologically stressful with widespread gene expression, cell signaling, inflammation, immune system activation, and elevated oxylipin production. This approach has been posited as advantageous for training adaptations, but there is little scientific support linking "training carbohydrate low" with improved exercise performance over the long term (62).

Polyphenol ingestion as a countermeasure to exercise-induced inflammation is receiving increasing attention by investigators $(62,63,66)$. Earlier studies reported few discernable benefits of increased polyphenol intake for athletes, but research design deficiencies portrayed a misunderstanding of polyphenol bioavailability and metabolism, effective dosing protocols, and appropriate outcome measures to capture bioactive effects (62). A recent study showed that adding 1 cup of blueberries per day for two weeks prior to a $75-\mathrm{km}$ cycling time trial strongly attenuated post-exercise plasma levels of 10 proinflammatory oxylipins (63). The cyclists ingesting blueberries experienced a 14 -fold variation, however, in plasma levels of 24 blueberry gut-derived metabolites following supplementation. The highest gut phenolic responders to blueberry intake experienced the lowest post-exercise plasma oxylipin levels. Little is known regarding the reasons for the high inter-individual variation in gut-derived metabolites after polyphenol ingestion. This may be related in part to differences in gut microbiota $\alpha$ diversity (richness) and differences in phase I and II metabolic enzymes and phase III transporters $(14,67)$. Whether or not the cyclists with a low gut-phenolic response to $1 \mathrm{cup} /$ day blueberry ingestion would benefit from doubling their intake is unknown.

The gut microbiota includes a large collection of bacteria, viruses, fungi, and archaea, and the richness or $\alpha$-diversity varies widely between individuals $(14,68)$. Plant-based dietary patterns and regular exercise training have an influence on the gut microbiota, increasing $\alpha$-diversity and the production of metabolites such as short chain fatty acids (SCFA) from dietary fiber and gut-derived phenolics from plant polyphenols $(14,68,69)$. SCFAs may support athletic performance by influencing fuel utilization and skeletal muscle function, and gut-derived phenolics may improve post-exercise metabolic recovery by mitigating inflammation (68). SCFAs are the preferred fuel for colonocytes, and have also been linked to regulation of energy homeostasis, body weight, immune function, and inflammation (69).

\section{THE FUTURE OF PRECISION SPORTS NUTRITION}

The metabolic variance in the way individuals respond to exercise and nutritional interventions is considerable and largely unexplained. The other major challenge in precision nutrition includes the translation of costly multiomics biomarker data to evidence-based individualized nutrition recommendations that are acceptable to the athlete and efficacious in terms of actual performance, recovery, and health.

At this time, a precision sports nutrition approach based on integrated multiomics to tailor recommendations at the individual athlete level is an emerging discipline with more questions than answers. Precision nutrition emphasizes multiomics tools and methods to sharpen the scientific certainty needed for specific nutrition recommendations for athletes and fitness enthusiasts. However, to make this work, larger studies are needed that focus on mechanisms underlying metabolic heterogeneity with deep phenotyping, multiomics, and machine learning (6). Thus, precision nutrition will require huge investments and scientific advances before this approach becomes accurate and practical for athletes. 
The costs and scientific challenges make this stratagem appear unattainable, but what is being accomplished today in precision nutrition seemed impossible just two decades ago.

\section{REFERENCES}

1. U.S. Department of Agriculture and U.S. Department of Health and Human Services. Dietary Guidelines for Americans, 2020-2025. 9th Edition. December 2020. Available online at: DietaryGuidelines.gov.

2. Adams SH, Anthony JC, Carvajal R, Chae L, Khoo CSH, Latulippe ME, et al. Perspective: guiding principles for the implementation of personalized nutrition approaches that benefit health and function. Adv Nutr. (2020) 11:25-34. doi: 10.1093/advances/nmz086

3. Zeisel SH. Precision (Personalized) Nutrition: understanding metabolic heterogeneity. Ann Rev Food Sci Technol. (2020) 11:71-92. doi: 10.1146/annurev-food-032519-051736

4. Gibney ER. Personalised nutrition - phenotypic and genetic variation in response to dietary intervention. Proc Nutr Soc. (2020) 79:23645. doi: 10.1017/S0029665119001137

5. Stover PJ, King JC. More nutrition precision, better decisions for the health of our nation. J Nutr. (2020) 150:3058-60. doi: 10.1093/jn/nxaa280

6. Morand C, De Roos B, Garcia-Conesa MT, Gibney ER, Landberg R, Manach $\mathrm{C}$, et al. Why interindividual variation in response to consumption of plant food bioactives matters for future personalised nutrition. Proc Nutr Soc. (2020) 79:225-35. doi: 10.1017/S0029665120000014

7. Kaput J. Developing the Pathway to Personalized Health: The potential of N-of-1 studies for personalizing nutrition. J Nutr. (2021) 151:28634. doi: $10.1093 /$ jn/nxab243

8. Floris M, Cano A, Porru L, Addis R, Cambedda A, Idda ML, et al. Direct-to-consumer nutrigenetics testing: an overview. Nutrients. (2020) 12:566. doi: 10.3390/nu12020566

9. Livingstone KM, Celis-Morales C, Navas-Carretero S, San-Cristobal R, Forster H, Woolhead C, et al. Food4Me study. Personalised nutrition advice reduces intake of discretionary foods and beverages: findings from the Food4Me randomised controlled trial. Int J Behav Nutr Phys Act. (2021) 18:70. doi: 10.1186/s12966-021-01136-5

10. Ordovas JM, Ferguson LR, Tai ES, Mathers JC. Personalised nutrition and health. BMJ. (2018) 361:bmj.k2173. doi: 10.1136/bmj.k2173

11. Moore JB. From personalised nutrition to precision medicine: the rise of consumer genomics and digital health. Proc Nutr Soc. (2020) 79:30010. doi: 10.1017/S0029665120006977

12. Brennan L, de Roos B. Nutrigenomics: lessons learned and future perspectives. Am J Clin Nutr. (2021) 113:503-16. doi: 10.1093/ajcn/nqaa366

13. Mullins VA, Bresette W, Johnstone L, Hallmark B, Chilton FH. Genomics in personalized nutrition: can you "eat for your genes"? Nutrients. (2020) 12:3118. doi: 10.3390/nu12103118

14. Asnicar F, Berry SE, Valdes AM, Nguyen LH, Piccinno G, Drew DA, et al. Microbiome connections with host metabolism and habitual diet from 1,098 deeply phenotyped individuals. Nat Med. (2021) 27:32132. doi: 10.1038/s41591-020-01183-8

15. Kirk D, Catal C, Tekinerdogan B. Precision nutrition: A systematic literature review. Comput Biol Med. (2021) 133:104365. doi: 10.1016/j.compbiomed.2021.104365

16. Bouchard C. Genetics of obesity: what we have learned over decades of research. Obesity. (2021) 29:802-20. doi: 10.1002/oby.23116

17. San-Cristobal R, Navas-Carretero S, Martínez-González MÁ, Ordovas JM, Martínez JA. Contribution of macronutrients to obesity: implications for precision nutrition. Nat Rev Endocrinol. (2020) 16:305-20. doi: 10.1038/s41574-020-0346-8

18. Maughan RJ, Burke LM, Dvorak J, Larson-Meyer DE, Peeling P, Phillips $\mathrm{SM}$, et al. IOC consensus statement: dietary supplements and the highperformance athlete. Int J Sport Nutr Exerc Metab. (2018) 28:10425. doi: 10.1123/ijsnem.2018-0020

\section{AUTHOR CONTRIBUTIONS}

DN wrote this manuscript and agrees to be accountable for the content of the work.

19. Peeling P, Castell LM, Derave W, de Hon O, Burke LM. Sports foods and dietary supplements for optimal function and performance enhancement in track-and-field athletes. Int J Sport Nutr Exerc Metab. (2019) 29:198209. doi: 10.1123/ijsnem.2018-0271

20. Thomas DT, Erdman KA, Burke LM. American College of Sports Medicine joint position statement nutrition and athletic performance. Med Sci Sports Exerc. (2016) 48:543-68. doi: 10.1249/MSS.0000000000000852

21. Burke LM, Castell LM, Casa DJ, Close GL, Costa RJS, Desbrow B, et al. International Association of Athletics Federations consensus statement 2019: nutrition for athletics. Int J Sport Nutr Exerc Metab. (2019) 29:7384. doi: 10.1123/ijsnem.2019-0065

22. Jeukendrup AE. Periodized nutrition for athletes. Sports Med. (2017) 47:5163. doi: 10.1007/s40279-017-0694-2

23. Conkright W, Deuster PA. Precision performance nutrition what can Special Operations Forces communities expect? J Spec Oper Med. (2019) 19:107-12.

24. Phillips AM. 'Only a click away - DTC genetics for ancestry, health, love... and more: a view of the business and regulatory landscape'. Appl Transl Genom. (2016) 8:16-22. doi: 10.1016/j.atg.2016.01.001

25. Webborn N, Williams A, McNamee M, Bouchard C, Pitsiladis Y, Ahmetov I, et al. Direct-to-consumer genetic testing for predicting sports performance and talent identification: consensus statement. Br J Sports Med. (2015) 49:148691. doi: 10.1136/bjsports-2015-095343

26. Vlahovich N, Fricker PA, Brown MA, Hughes D. Ethics of genetic testing and research in sport: a position statement from the Australian Institute of Sport. Br J Sports Med. (2017) 51:5-11. doi: 10.1136/bjsports-2016-096661

27. Ghosh S, Hota M, Chai X, Kiranya J, Ghosh P, He Z, et al. Exploring the underlying biology of intrinsic cardiorespiratory fitness through integrative analysis of genomic variants and muscle gene expression profiling. J Appl Physiol. (2019) 126:1292-314. doi: 10.1152/japplphysiol.00035.2018

28. Zhao Y, Huang G, Chen Z, Fan X, Huang T, Liu J, et al. Four loci are associated with cardiorespiratory fitness and endurance performance in young Chinese females. Sci Rep. (2020) 10:10117. doi: 10.1038/s41598-020-67045-y

29. Williams CJ, Williams MG, Eynon N, Ashton KJ, Little JP, Wisloff U, et al. Genes to predict VO2max trainability: a systematic review. BMC Genomics. (2017) 18:831. doi: 10.1186/s12864-017-4192-6

30. Barber JL, Ruiz-Ramie JJ, Robbins JM, Gerszten RE, Leon AS, Rao DC, et al. Regular exercise and patterns of response across multiple cardiometabolic traits: the HERITAGE family study. Br J Sports Med. (2021) 35:222231. doi: 10.1136/bjsports-2020-103323

31. Guest NS, Horne J, Vanderhout SM, El-Sohemy A. Sport nutrigenomics: personalized nutrition for athletic performance. Front Nutr. (2019) 6:8. doi: 10.3389/fnut.2019.00008

32. Castro A, Duft RG, Silva LM, Ferreira MLV, Andrade ALL, Bernardes CF, et al. Understanding the relationship between intrinsic cardiorespiratory fitness and serum and skeletal muscle metabolomics profile. J Proteome Res. (2021) 20:2397-409. doi: 10.1021/acs.jproteome.0c00905

33. Robbins JM, Peterson B, Schranner D, Tahir UA, Rienmüller T, Deng S, et al. Human plasma proteomic profiles indicative of cardiorespiratory fitness. Nat Metab. (2021) 3:786-97. doi: 10.1038/s42255-021-00400-z

34. Grgic J, Grgic I, Pickering C, Schoenfeld BJ, Bishop DJ, Pedisic Z. Wake up and smell the coffee: caffeine supplementation and exercise performancean umbrella review of 21 published meta-analyses. Br J Sports Med. (2020) 54:681-8. doi: 10.1136/bjsports-2018-100278

35. Barreto G, Grecco B, Merola P, Reis CEG, Gualano B, Saunders B. Novel insights on caffeine supplementation, CYP1A2 genotype, physiological responses and exercise performance. Eur J Appl Physiol. (2021) 121:74969. doi: 10.1007/s00421-020-04571-7

36. Grgic J, Pickering C, Del Coso J, Schoenfeld BJ, Mikulic P. CYP1A2 genotype and acute ergogenic effects of caffeine intake 
on exercise performance: a systematic review. Eur J Nutr. (2021) 60:1181-95. doi: 10.1007/s00394-020-02427-6

37. Zeisel SH, Klatt KC, Caudill MA. Choline. Adv Nutr. (2018) 9:5860. doi: 10.1093/advances/nmx004

38. Moretti A, Paoletta M, Liguori S, Bertone M, Toro G, Iolascon G. Choline: an essential nutrient for skeletal muscle. Nutrients. (2020) 12:2144. doi: 10.3390/nu12072144

39. da Costa KA, Corbin KD, Niculescu MD, Galanko JA, Zeisel SH. Identification of new genetic polymorphisms that alter the dietary requirement for choline and vary in their distribution across ethnic and racial groups. FASEB J. (2014) 28:2970-8. doi: 10.1096/fj.14-249557

40. da Costa KA, Badea M, Fischer LM, Zeisel SH. Elevated serum creatine phosphokinase in choline-deficient humans: mechanistic studies in C2C12 mouse myoblasts. Am J Clin Nutr. (2004) 80:163-70. doi: 10.1093/ajcn/80.1.163

41. Kohlmeier M, da Costa KA, Fischer LM, Zeisel SH. Genetic variation of folate-mediated one-carbon transfer pathway predicts susceptibility to choline deficiency in humans. Proc Natl Acad Sci U S A. (2005) 102:1602530. doi: 10.1073/pnas.0504285102

42. Deuster PA, Singh A, Coll R, Hyde DE, Becker WJ. Choline ingestion does not modify physical or cognitive performance. Mil Med. (2002) 167:10205. doi: 10.1093/milmed/167.12.1020

43. Warber JP, Patton JF, Tharion WJ, Zeisel SH, Mello RP, Kemnitz CP, et al. The effects of choline supplementation on physical performance. Int J Sport Nutr Exerc Metab. (2000) 10:170-81. doi: 10.1123/ijsnem.10.2.170

44. Das SK, Miki AJ, Blanchard CM, Sazonov E, Gilhooly CH, Dey S, et al. Perspective: opportunities and challenges of technology tools in dietary and activity assessment: bridging stakeholder viewpoints. Adv Nutr. (2021) 20:nmab103. doi: 10.1093/advances/nmab103

45. Nieman DC. Nutritional Assessment. (7 ${ }^{\text {th }}$ ed.) New York, NY: McGraw Hill, (2019).

46. Brunyé TT, Yau K, Okano K, Elliott G, Olenich S, Giles GE, et al. Toward predicting human performance outcomes from wearable technologies: a computational modeling approach. Front Physiol. (2021) 12:1499. doi: 10.3389/fphys.2021.738973

47. Mortazavi BJ, Gutierrez-Osuna R, A. review of digital innovations for diet monitoring and precision nutrition. J Diabetes Sci Technol. (2021) 1:19322968211041356. doi: 10.1177/19322968211041356

48. Dunn J, Kidzinski L, Runge R, Witt D, Hicks JL, SchüsslerFiorenza Rose SM, et al. Wearable sensors enable personalized predictions of clinical laboratory measurements. Nat Med. (2021) 27:1105-12. doi: 10.1038/s41591-021-01339-0

49. Kim DS, Wheeler MT, Ashley EA. The genetics of human performance. Nat Rev Genet. (2021) 14:1-5. doi: 10.1038/s41576-021-00400-5

50. Contrepois K, Wu S, Moneghetti KJ, Hornburg D, Ahadi S, Tsai MS, et al. Molecular choreography of acute exercise. Cell. (2020) 181:111230.e16. doi: 10.1016/j.cell.2020.04.043

51. Morville T, Sahl RE, Moritz T, Helge JW, Clemmensen C. Plasma metabolome profiling of resistance exercise and endurance exercise in humans. Cell Rep. (2020) 33:108554. doi: 10.1016/j.celrep.2020.108554

52. Amar D, Lindholm ME, Norrbom J, Wheeler MT, Rivas MA, Ashley EA. Time trajectories in the transcriptomic response to exercise - a meta-analysis. Nat Commun. (2021) 12:3471. doi: 10.1038/s41467-021-23579-x

53. Sakaguchi CA, Nieman DC, Signini EF, Abreu RM, Catai AM. Metabolomics-based studies assessing exercise-induced alterations of the human metabolome: a systematic review. Metabolites. (2019) 9:164. doi: 10.3390/metabo9080164

54. Nieman DC, Groen AJ, Pugachev A, Vacca G. Detection of functional overreaching in endurance athletes using proteomics. Proteomes. (2018) 6:33. doi: 10.3390/proteomes6030033

55. Nieman DC, Groen AJ, Pugachev A, Simonson AJ, Polley K, James $\mathrm{K}$, et al. Proteomics-based detection of immune dysfunction in an elite adventure athlete trekking across the Antarctica. Proteomes. (2020) 8:4. doi: 10.3390/proteomes 8010004

56. Nayor M, Shah RV, Miller PE, Blodgett JB, Tanguay M, Pico $\mathrm{AR}$, et al. Metabolic architecture of acute exercise response in middle-aged adults in the community. Circulation.

$(2020)$ 142:1905-24. doi: 10.1161/CIRCULATIONAHA.120.050281

57. Signini ÉF, Nieman DC, Silva CD, Sakaguchi CA, Catai AM. Oxylipin response to acute and chronic exercise: a systematic review. Metabolites. (2020) 10:264. doi: 10.3390/metabo1006 0264

58. Nieman DC, Gillitt ND, Sha W. Identification of a select metabolite panel for measuring metabolic perturbation in response to heavy exertion. Metabolomics. (2018) 14:147. doi: 10.1007/s11306-018-1444-7

59. Nieman DC, Pence BD. Exercise immunology: Future directions. J Sport Health Sci. (2020) 9:432-45. doi: 10.1016/j.jshs.2019.1 2.003

60. Whitham M, Parker BL, Friedrichsen M, Hingst JR, Hjorth M, Hughes WE, et al. Extracellular vesicles provide a means for tissue crosstalk during exercise. Cell Metab. (2018) 27:237-51.e4. doi: 10.1016/j.cmet.2017.12.001

61. Sanford JA, Nogiec CD, Lindholm ME, Adkins JN, Amar D, Dasari S, et al. Molecular Transducers of Physical Activity Consortium (MoTrPAC): mapping the dynamic responses to exercise. Cell. (2020) 181:146474. doi: 10.1016/j.cell.2020.06.004

62. Nieman DC, Lila MA, Gillitt ND. Immunometabolism: a multiomics approach to interpreting the influence of exercise and diet on the immune system. Annu Rev Food Sci Technol. (2019) 10:341-63. doi: 10.1146/annurev-food-032818-121316

63. Nieman DC, Gillitt ND, Chen GY, Zhang Q, Sha W, Kay CD, et al. Blueberry and/or banana consumption mitigate arachidonic, cytochrome P450 oxylipin generation during recovery from $75-\mathrm{km}$ cycling: a randomized trial. Front Nutr. (2020) 7:121. doi: 10.3389/fnut.2020.00121

64. Nieman DC, Gillitt ND, Chen GY, Zhang Q, Sakaguchi CA, Stephan EH. Carbohydrate intake attenuates post-exercise plasma levels of cytochrome P450-generated oxylipins. PLOS ONE. (2019) 14:e0213676. doi: 10.1371/journal.pone.0213676

65. Nieman DC, Gillitt ND, Sha W, Esposito D, Ramamoorthy S. Metabolic recovery from heavy exertion following banana compared to sugar beverage or water only ingestion: A randomized, crossover trial. PLoS One. (2018) 13:e0194843. doi: 10.1371/journal.pone.0194843

66. Jantan I, Haque MA, Arshad L, Harikrishnan H, Septama AW, MohamedHussein ZA. Dietary polyphenols suppress chronic inflammation by modulation of multiple inflammation-associated cell signaling pathways. Nutr Biochem. (2021) 93:108634. doi: 10.1016/j.jnutbio.2021.108634

67. Cladis DP, Simpson AMR, Cooper KJ, Nakatsu CH, Ferruzzi MG, Weaver CM. Blueberry polyphenols alter gut microbiota \& phenolic metabolism in rats Food Funct. (2021) 12:2442-56. doi: 10.1039/D0FO03457F

68. Hughes RL, Holscher HD. Fueling gut microbes: a review of the interaction between diet, exercise, and the gut microbiota in athletes. Adv Nutr. (2021) 6:nmab077. doi: 10.1093/advances/nmab077

69. Alexander C, Swanson KS, Fahey GC, Garleb KA. Perspective: physiologic importance of short-chain fatty acids from nondigestible carbohydrate fermentation. Adv Nutr. (2019) 10:576-89. doi: 10.1093/advances/nmz004

Conflict of Interest: The author declares that the research was conducted in the absence of any commercial or financial relationships that could be construed as a potential conflict of interest.

Publisher's Note: All claims expressed in this article are solely those of the authors and do not necessarily represent those of their affiliated organizations, or those of the publisher, the editors and the reviewers. Any product that may be evaluated in this article, or claim that may be made by its manufacturer, is not guaranteed or endorsed by the publisher.

Copyright (C) 2021 Nieman. This is an open-access article distributed under the terms of the Creative Commons Attribution License (CC BY). The use, distribution or reproduction in other forums is permitted, provided the original author(s) and the copyright owner(s) are credited and that the original publication in this journal is cited, in accordance with accepted academic practice. No use, distribution or reproduction is permitted which does not comply with these terms. 